\title{
Simplified Method of Evaluating Integrals of Powers of Sine Using Reduction Formula as Mathematical Algorithm
}

\author{
Lito E. Suello* \\ Malayan Colleges Laguna, Pulo-Diezmo Road, Cabuyao City, Laguna, Philippines. \\ * Corresponding author. Tel.: +639497688588; email: lesuello@mcl.edu.ph \\ Manuscript submitted January 25, 2015; accepted June 5, 2015. \\ doi: 10.17706/ijapm.2015.5.3.192-198
}

\begin{abstract}
This paper presents a simpler and shorter method of evaluating integrals of powers of sine. The reduction formula for sine is repeatedly applied to the integral of the nth power of sine until generalized formulas are derived. Since the derivation process involves recursive relations, the coefficients and exponents of the derived formulas showed certain patterns and sequences which were used as the basis for developing an easier algorithm.
\end{abstract}

Key words: Integration, mathematical algorithm, powers of sine, reduction formula, trigonometric identities.

\section{Introduction}

Evaluating integrals of powers of trigonometric functions is always part of the study of Integral Calculus. Integrals of powers of sine are usually evaluated using trigonometric identities and the solution depends on whether the power is odd or even. For odd powers, the integrand is transformed by factoring out one sine and the remaining even powered sine is converted into cosine using the identity $\sin ^{2} x=1-\cos ^{2} x$. The integral is then evaluated using power formula with the factored sine used as the differential of cosine. For even powers, the double angle identity $\sin ^{2} x=\frac{1}{2}(1-\cos 2 x)$ is used to reduce the power of sine into an expression where direct integration formulas can already be applied [1]-[3].

Another method used to evaluate powers of sine is by using reduction formula. A reduction formula transforms the integral into an integral of the same or similar expression with a lower integer exponent [4]. It is repeatedly applied until the power of the last term is reduced to two or one, and the final integral can be evaluated. Using integration by parts, the reduction formula for sine is [5].

$$
\int \sin ^{n} a x d x=-\frac{1}{a(n)} \sin ^{n-1} a x \cos a x+\frac{n-1}{n} \int \sin ^{n-2} a x d x
$$

The methods discussed above are normally tedious and time consuming depending on the given power of sine. As shown in the study of Dampil [6], deriving generalized formulas can simplify solutions, hence, the objective of this paper is to come up with a shorter and simpler method of integrating powers of sine. Generalized formulas are derived by successive application of the reduction formula to the integral of the nth power of sine. Because of the recursive nature of the reduction formula, an algorithm is developed 
based on the sequences and patterns of the coefficients and exponents of the terms of the derived formulas.

\section{Derivation of Formulas}

Given: $\int \sin ^{n} a x d x$, where $n$ is any integer

Using the reduction formula,

$$
\int \sin ^{n} \operatorname{axd} x=-\frac{1}{a(n)} \sin ^{n-1} \operatorname{axcos} a x+\frac{n-1}{n} \int \sin ^{n-2} a x d x
$$

Applying the reduction formula to the last term

$$
\int \sin ^{n} a x d x=-\frac{1}{a(n)} \sin ^{n-1} \operatorname{axcos} a x+\frac{n-1}{n}\left[-\frac{1}{a(n-2)} \sin ^{n-3} \operatorname{axcos} a x+\frac{n-3}{n-2} \int \sin ^{n-4} a x d x\right]
$$

Applying the reduction formula again,

$$
\begin{gathered}
\int \sin ^{n} \operatorname{axd} d x=-\frac{1}{a(n)} \sin ^{n-1} \operatorname{axcos} a x-\frac{n-1}{a(n)(n-2)} \sin ^{n-3} \operatorname{axcos} a x- \\
\frac{(n-1)(n-3)}{n(n-2)}\left[-\frac{1}{a(n-4)} \sin ^{n-5} \operatorname{axcos} a x+\frac{n-3}{n-4} \int \sin ^{n-6} a x d x\right]
\end{gathered}
$$

Simplifying,

$$
\begin{aligned}
& \int \sin ^{n} a x d x=-\frac{1}{a(n)} \sin ^{n-1} \operatorname{axcos} a x-\frac{n-1}{a(n)(n-2)} \sin ^{n-3} a x \cos a x- \\
& \frac{(n-1)(n-3)}{a(n)(n-2)(n-4)} \sin ^{n-5} \operatorname{axcos} a x+\frac{(n-1)(n-3)}{n(n-2)(n-4)} \int \sin ^{n-6} a x d x
\end{aligned}
$$

The same trend continues until the last term becomes

$\int \sin \operatorname{axd} x$ if $n$ is odd, or

$\int \sin ^{2} a x d x$ if $n$ is even

\subsection{Odd Powers}

$$
\begin{gathered}
\int \sin ^{n} a x d x=-\frac{1}{a(n)} \sin ^{n-1} \operatorname{axcos} a x-\frac{n-1}{a(n)(n-2)} \sin ^{n-3} \operatorname{axcos} a x- \\
\frac{(n-1)(n-3)}{a(n)(n-2)(n-4)} \sin ^{n-5} \operatorname{axcos} a x-\ldots .+\frac{(n-1)(n-3)(n-5) \ldots(2)}{n(n-2)(n-4)(n-6) \ldots(3)} \int \sin a x d x
\end{gathered}
$$

Integrating the last term,

$$
\int \sin ^{n} a x d x=-\frac{1}{a(n)} \sin ^{n-1} a x \cos a x-\frac{n-1}{a(n)(n-2)} \sin ^{n-3} a x \cos a x-\frac{n-1}{a(n)(n-2)} \sin ^{n-3} a x \cos a x-
$$




$$
\frac{(n-1)(n-3)}{a(n)(n-2)(n-4)} \sin ^{n-5} a x \cos a x-\ldots . .-\frac{(n-1)(n-3)(n-5) \ldots .(2)}{a(n)(n-2)(n-4)(n-6) \ldots(3)} \cos a x+C
$$

Factoring out the common factor gives the formula,

$$
\begin{gathered}
\int \sin ^{n} a x d x=-\frac{\cos a x}{a}\left[\frac{1}{n} \sin ^{n-1} a x+\frac{n-1}{n(n-2)} \sin ^{n-3} a x+\frac{(n-1)(n-3)}{n(n-2)(n-4)} \sin ^{n-5} a x+\ldots .+\right. \\
\left.\frac{(n-1)(n-3)(n-5) \ldots(2)}{n(n-2)(n-4)(n-6) \ldots(3)}\right]+C
\end{gathered}
$$

It can also be written as

$$
\int \sin ^{n} a x d x=-\frac{\cos a x}{a}\left[C_{0} \sin ^{n-1} a x+\sum_{j=1}^{\frac{n-1}{2}} C_{j} \sin ^{n-2 j-1} a x\right]+C
$$

where: $C_{0}=\frac{1}{n}$, and $C_{j}=C_{j-1}\left[\frac{n-2 j+1}{n-2 j}\right]$

\subsection{Even Powers}

$$
\begin{gathered}
\int \sin ^{n} a x d x=-\frac{1}{a(n)} \sin ^{n-1} \operatorname{axcos} a x-\frac{n-1}{a(n)(n-2)} \sin ^{n-3} a x \cos a x- \\
\frac{(n-1)(n-3)}{a(n)(n-2)(n-4)} \sin ^{n-5} \operatorname{axcos} a x-\ldots .+\frac{(n-1)(n-3)(n-5) \ldots(3)}{n(n-2)(n-4)(n-6) \ldots(4)} \int \sin ^{2} a x d x
\end{gathered}
$$

Applying the reduction formula to the last term,

$$
\begin{aligned}
\int \sin ^{n} a x d x=- & \frac{1}{a(n)} \sin ^{n-1} \operatorname{axcos} a x-\frac{n-1}{a(n)(n-2)} \sin ^{n-3} \operatorname{axcos} a x-\frac{(n-1)(n-3)}{a(n)(n-2)(n-4)} \sin ^{n-5} \operatorname{axcos} a x \\
& -\ldots .+\frac{(n-1)(n-3)(n-5) \ldots(3)}{n(n-2)(n-4)(n-6) \ldots(4)}\left[-\frac{1}{a(2)} \sin a x \cos a x+\frac{1}{2} \sin ^{0} a x d x\right]
\end{aligned}
$$

Simplifying,

$$
\begin{aligned}
\int \sin ^{n} a x d x= & -\frac{1}{a(n)} \sin ^{n-1} a x \cos a x-\frac{n-1}{a(n)(n-2)} \sin ^{n-3} \operatorname{axcos} a x-\frac{(n-1)(n-3)}{a(n)(n-2)(n-4)} \sin ^{n-5} \operatorname{axcos} a x \\
& -\ldots-\frac{(n-1)(n-3) \ldots(3)}{a(n)(n-2)(n-4) \ldots(2)} \sin a x \cos a x+\frac{(n-1)(n-3) \ldots(3)}{a(n)(n-2)(n-4) \ldots(2)} x+C
\end{aligned}
$$

Factoring out the common factor gives,

$$
\int \sin ^{n} a x d x=-\frac{\cos a x}{a}\left[\frac{1}{n} \sin ^{n-1} a x+\frac{n-1}{n(n-2)} \sin ^{n-3} a x+\frac{(n-1)(n-3)}{n(n-2)(n-4)} \sin ^{n-5} a x+\ldots+\right.
$$




$$
\left.\frac{(n-1)(n-3)(n-5) \ldots(3)}{n(n-2)(n-4)(n-6) \ldots(2)} \sin a x\right]+\frac{(n-1)(n-3)(n-5) \ldots(3)}{n(n-2)(n-4)(n-6) \ldots(2)} x+C
$$

The formula may also be written as,

$$
\int \sin ^{n} a x d x=-\frac{\cos a x}{a}\left[C_{0} \sin ^{n-1} a x+\sum_{j=1}^{\frac{n-2}{2}} C_{j} \sin ^{n-2 j-1} a x\right]+C_{\frac{n-2}{2}} x+C
$$

where: $C_{0}=\frac{1}{n}$, and $C_{j}=C_{j-1}\left[\frac{n-2 j+1}{n-2 j}\right]$

\section{Development of the Algorithm for the New Method}

A simpler and easier procedure can be developed from the observed trends of the coefficients and exponents of the derived formulas. These are summarized as follows:

\subsection{Odd Powers}

- Write $-\frac{\cos a x}{a}$. This will be followed by a series of sine terms. For example, $\int \sin ^{5} 2 x d x$

$$
-\frac{\cos 2 x}{2}
$$

- The first term of the series has a coefficient of $\frac{1}{n}$ and the exponent of sine is $n-1$. This coefficient and exponent will be used in determining the coefficient and exponent of the next term.

$$
-\frac{\cos 2 x}{2}\left[\frac{1}{5} \sin ^{4} 2 x+\right.
$$

- For the next term, the coefficient has a numerator equal to the product of the exponent and the numerator of the preceding term. The denominator is the product of the denominator and exponent minus one of the preceding term. The exponent of sine is the exponent of the preceding term minus two.

$$
-\frac{\cos 2 x}{2}\left[\frac{1}{5} \sin ^{4} 2 x+\frac{(1)(4)}{(5)(3)} \sin ^{2} 2 x\right.
$$

- Follow the same procedure until the exponent of sine becomes zero which terminates the series.

$$
-\frac{\cos 2 x}{2}\left[\frac{1}{5} \sin ^{4} 2 x+\frac{4}{15} \sin ^{2} 2 x+\frac{(4)(2)}{(15)(!)} \sin ^{0} 2 x\right]
$$

- Add a constant of integration.

$$
\int \sin ^{5} 2 x d x=-\frac{\cos 2 x}{2}\left[\frac{1}{5} \sin ^{4} 2 x+\frac{4}{15} \sin ^{2} 2 x+\frac{5}{15}\right]+C
$$




\subsection{Even Powers}

- Write $-\frac{\cos a x}{a}$. This will be followed by a series of sine terms. For example, $\int \sin ^{6} 3 x d x$

$$
-\frac{\cos 3 x}{3}
$$

- The first term of the series has a coefficient of $\frac{1}{n}$ and the exponent of sine is n-1. This coefficient and exponent will be used in determining the coefficient and exponent of the next term.

$$
-\frac{\cos 3 x}{3}\left[\frac{1}{6} \sin ^{5} 3 x+\right.
$$

- For the next term, the coefficient has a numerator equal to the product of the exponent and the numerator of the preceding term. The denominator is the product of the denominator and exponent minus one of the preceding term. The exponent of sine is the exponent of the preceding term minus two.

$$
-\frac{\cos 3 x}{3}\left[\frac{1}{6} \sin ^{5} 3 x+\frac{(1)(5)}{(6)(4)} \sin ^{3} 3 x+\right.
$$

- Follow the same procedure until the exponent of sine becomes one which terminates the series.

$$
-\frac{\cos 3 x}{3}\left[\frac{1}{6} \sin ^{5} 3 x+\frac{5}{24} \sin ^{3} 3 x+\frac{(5)(3)}{(24)(2)} \sin ^{1} 3 x\right]
$$

- The next term is the product of $x$ and the coefficient of the last term in the sine series.

$$
-\frac{\cos 3 x}{3}\left[\frac{1}{6} \sin ^{5} 3 x+\frac{5}{24} \sin ^{3} 3 x+\frac{15}{48} \sin 3 x\right]+\frac{15}{48} x
$$

- Add a constant of integration.

$$
\int \sin ^{6} 3 x d x=-\frac{\cos 3 x}{3}\left[\frac{1}{6} \sin ^{5} 3 x+\frac{5}{24} \sin ^{3} 3 x+\frac{15}{48} \sin 3 x\right]+\frac{15}{48} x+C
$$

\section{Comparison between the Old and the New Method}

Evaluate $\int \sin ^{7} 4 x d x$

Using the Old Method

$$
\int \sin ^{7} 5 x d x=-\frac{1}{5(7)} \sin ^{6} 5 x \cos 5 x+\frac{6}{7} \int \sin ^{5} 5 x d x
$$




$$
\begin{gathered}
=-\frac{1}{35} \sin ^{6} 5 x \cos 5 x+\frac{6}{7}\left[-\frac{1}{5(5)} \sin ^{4} 5 x \cos 5 x+\frac{4}{5} \int \sin ^{3} 5 x\right] \\
=-\frac{1}{35} \sin ^{6} 5 x \cos 5 x-\frac{6}{175} \sin ^{4} 5 x \cos 5 x+\frac{24}{35}\left[-\frac{1}{5(3)} \sin ^{2} 5 x \cos 5 x+\frac{2}{3} \int \sin 5 x d x\right] \\
=-\frac{1}{35} \sin ^{6} 5 x \cos 5 x-\frac{6}{175} \sin ^{4} 5 x \cos 5 x-\frac{24}{525} \sin ^{2} 5 x \cos 5 x-\frac{48}{525} \cos 5 x+C \\
=-\frac{\cos 5 x}{5}\left[\frac{1}{7} \sin ^{6} 5 x+\frac{6}{35} \sin ^{4} 5 x+\frac{24}{105} \sin ^{2} 5 x+\frac{48}{105}\right]+C
\end{gathered}
$$

Using the New Method

$$
\begin{gathered}
\int \sin ^{7} 5 x d x=-\frac{\cos 5 x}{5}\left[\frac{1}{7} \sin ^{6} 5 x+\frac{(1)(6)}{(7)(5)} \sin ^{4} 5 x+\frac{(6)(4)}{(35)(3)} \sin ^{2} 5 x+\frac{(24)(2)}{(105)(1)} \sin ^{0} 5 x\right]+C \\
\int \sin ^{7} 5 x d x=-\frac{\cos 5 x}{5}\left[\frac{1}{7} \sin ^{6} 5 x+\frac{6}{35} \sin ^{4} 5 x+\frac{24}{105} \sin ^{2} 5 x+\frac{48}{105}\right]+C
\end{gathered}
$$

Evaluate $\int \sin ^{4} 2 x$

Using the Old Method

$$
\begin{gathered}
\int \sin ^{4} 2 x d x=-\frac{1}{2(4)} \sin ^{3} 2 x \cos 2 x+\frac{3}{4} \int \sin ^{2} 2 x d x \\
\int \sin ^{4} 2 x d x=-\frac{1}{8} \sin ^{3} 2 x \cos 2 x+\frac{3}{4}\left[-\frac{1}{2(2)} \sin 2 x \cos 2 x+\frac{1}{2} \int d x\right] \\
\int \sin ^{4} 2 x d x=-\frac{1}{8} \sin ^{3} 2 x \cos 2 x-\frac{3}{16} \sin 2 x \cos 2 x+\frac{3}{8} x+C \\
\int \sin ^{4} 2 x d x=-\frac{\cos 2 x}{2}\left[\frac{1}{4} \sin ^{3} 2 x+\frac{3}{8} \sin 2 x\right]+\frac{3}{8} x+C
\end{gathered}
$$

Using the New Method

$$
\begin{gathered}
\int \sin ^{4} 2 x d x=-\frac{\cos 2 x}{2}\left[\frac{1}{4} \sin ^{3} 2 x+\frac{(1)(3)}{(4)(2)} \sin 2 x\right]+\frac{3}{8} x+C \\
\int \sin ^{4} 2 x d x=-\frac{\cos 2 x}{2}\left[\frac{1}{4} \sin ^{3} 2 x+\frac{3}{8} \sin 2 x\right]+\frac{3}{8} x+C
\end{gathered}
$$

\section{Conclusion}


The new method is simpler and easier to use since the tiresome repetitions of applying the reduction formula, or expansions of identities using the conventional methods are eliminated. Integrals can be evaluated directly since the procedure simply involves coefficients and exponents. It is very helpful since integrals of powers of sine are always encountered in higher mathematics courses like Differential Equations and Advanced Engineering Mathematics, and even in physics and mechanics. It can also be used in many engineering applications specifically in electricity and magnetism, waves, heat and mass transfer, and reaction kinetics. It is also suggested that the method be extended to integrals of powers of other trigonometric functions.

\section{Acknowledgment}

The author is very grateful to Susie and Bing Domingo for their support for the publication of this paper. He is likewise thankful to Engr. Fe G. Dampil for her encouragement and for being a model for aspiring researchers. He dedicates this paper to his wife Consuelo and daughter Victoria. Above all, he offers this endeavor to Almighty God for His faithfulness, wisdom, and unfailing love.

\section{References}

[1] Stewart, J. (2012). Calculus: Concepts and Contexts (4th ed.). ch. 5, pp. 389-390. Cengage.

[2] Hass, J., Weir, M., \& Thomas, G. (2014). University Calculus, ch. 8, pp. 439-440. Pearson.

[3] Varberg, D., Purcell, E., \& Rigdon, S. (2014). Calculus Early Transcendentals (1st ed.). ch. 7, pp. 385-386. Pearson.

[4] Riley, K. F., Hobson, M. P., \& Bence, S. J. (2010). Mathematical Methods for Physics and Engineering. Cambridge University Press.

[5] Stewart, J. (2014). Calculus: Early Transcendentals (7th ed.). ch. 7, pp. 467-468. Cengage.

[6] Dampil, F. (2014, May). Development of trigonometric formula for $\sin ^{4} n \theta$ and $\cos ^{4} n \theta$ using half-angle identities as mathematical algorithm. International Journal of applied Physics and Mathematics, 4(3), 155-158.

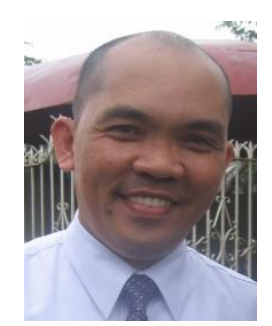

Lito E. Suello is a member of the Mathematical Society of the Philippines and the Philippine Institute of Chemical Engineers. He was born in Camarines Sur, Philippines on February 12, 1966. He is a licensed chemical engineer who finished his chemical engineering degree from Central Philippine University in Iloilo City, Philippines in 1987. He also completed his master in business administration from San Pedro College of Business Administration, Laguna, Philippines in 2006. At present, he is connected with Malayan Colleges Laguna, Philippines where he teaches mathematics, mechanics, and chemical engineering courses. 